\title{
Acute onset of inflammatory colitis in overlap with psoriatic arthritis and systemic lupus erythematosus following treatment with secukinumab
}

\author{
Sven Almer ${ }^{1,2}$, Francesca Faustini ${ }^{3,4}$, Sylwester Szczegielniak ${ }^{1,2}$ and Iva Gunnarsson ${ }^{3,4 *}$ \\ ${ }^{1}$ Department of Medicine, Solna, Karolinska Institutet, Sweden \\ ${ }^{2}$ Division of Gastroenterology, Karolinska University Hospital, Stockholm, Sweden \\ ${ }^{3}$ Department of Medicine, Division of Rheumatology Karolinska Institutet, Sweden \\ ${ }^{4}$ Rheumatology Karolinska, University Hospital, Stockholm, Sweden
}

A male diagnosed with psoriasis and psoriatic arthritis at age 18, underwent a kidney biopsy at age 23 due to persistent proteinuria, with findings of an immune-complex mediated mesangiocapillary nephritis with substantial glomerular sclerosis. Antinuclear antibodies (ANA) were negative but increased levels of antibodies against cardiolipin and $\gamma$ 2glycoprotein-I were detected.

At age 25, he presented acutely with fever and increased serum creatinine. A second kidney biopsy showed findings compatible with SLE nephritis class II but the diagnosis of SLE was not established as ANA was negative. Oral prednisolone and angiotensin II receptor blockers were given.

At age 26, he had spread psoriatic lesions and arthralgias, no arthritis was evident. ANA had become positive, high titers antiphospholipid antibodies remained. Complement factor 4 was decreased and he was now lymphopenic, Glomerular filtration was decreased $(61 \mathrm{ml} / \mathrm{min})$, urine albumin/creatinine ratio was increased $(162 \mathrm{mg} / \mathrm{mmol})$. Now fulfilling the classification criteria for SLE [1], hydroxychloroquine was added.

A year later, articular symptoms and skin psoriasis had worsened. HCQ had been withdrawn and treatment escalation was considered. TNF-inhibitors were regarded as contraindicated due to diagnosis of SLE and antiphospholipid antibody positivity, therefore he was started on subcutaneous secukinumab. Following the first injection, he promptly developed bowel symptoms, significant weight loss and urgency with 10-15 non-bloody daily bowel movements. Secukinumab was stopped after five injections.

A colonoscopy performed at 6 weeks from secukinumab start showed a moderately active total colitis with diffuse erythema, distorted and increased vascular pattern in the colon (Figure 1A). Histology showed crypt abscesses, cryptitis, and features of chronic inflammation. Granulomas were focally present in the colon and rectum. The findings were considered to be compatible with Crohn's disease. Treatment with prednisolone was increased and mesalazine was added.

Five months after secukinumab start, the bowel symptoms had improved slightly but high prednisolone doses were still required. A new colonoscopy revealed features of moderately active colitis; histopathology confirmed active colitis with pronounced inflammatory activity and multiple granulomas.
Due to the steroid-dependent inflammatory bowel activity, azathioprine was added but the gastrointestinal symptoms remained largely unaffected. A new renal biopsy was performed due to occurrence of urinary casts, showing a stable class II nephritis without signs of thrombotic microangiopathy.

A third colonoscopy at 15 months after secukinimab withdrawal was macroscopically normal without signs of active inflammation (Figure 1B). The bowel symptoms had markedly improved, but he was still unable to work.

With the aim of improving skin, joints symptoms and stabilizing the bowel long-term, treatment with intravenous ustekinumab was initiated 17 months after colitis onset. The effect on bowel symptoms and psoriatic lesions was prompt, and maintenance subcutaneous ustekinumab treatment was continued for now three years, a period during which he has been corticosteroid- free, regained weight, and taken up his full-time work. There were no relapses in the colitis or psoriatic lesions. Renal function has been stable, the albumin/creatinine ratio is $82 \mathrm{mg} / \mathrm{mmol}$ but there is still signs of complement activation.

New onset IBD or unraveling of pre-existing IBD under IL-17 inhibitors is a concern raised in some clinical trials [2] however the risk was not found to be elevated in a recent meta-analysis from treatment of inflammatory joint diseases [3]. Despite no history of bowel symptoms, granuloma formation was here detected at histological examination already early after treatment start, indicating uncovering of an already pre-existing, subclinical IBD. Since prior investigations were lacking and considering the known links between spondyloarthritides and intestinal inflammation [4], we cannot exclude this possibility.

IL-17 and IL-23 levels are increased in the intestinal mucosa of patients with IBD $[5,6]$, however, blocking of IL-17A leads to detrimental effect in IBD [7] as IL-17A seems crucial in maintaining the intestinal homeostasis and integrity of the enterocyte epithelial barrier [8].

${ }^{\star}$ Correspondence to: Iva Gunnarsson, Department of Medicine, Division of Rheumatology Karolinska Institutet, Solna, Unit of Rheumatology, Karolinska University Hospital, Stockholm, Sweden, E-mail: iva.gunnarsson@ki.se

Received: December 28, 2020; Accepted: January 05, 2021; Published: January 09, 2021 

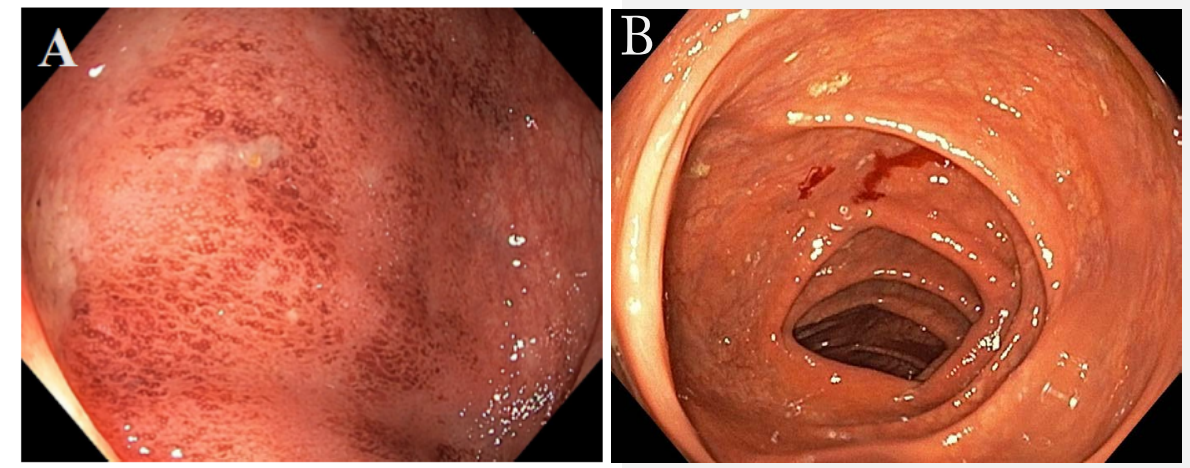

Figure 1. Colonoscopy findings 6 weeks after initiation of secukinumab treatment in a 27- years old man with psoriatic arthritis and systemic lupus erythematosus. (A) Presence of moderately active colitis in the ascending colon at baseline (B) and improvement after 15 months

Withdrawal of secukinumab was here followed by a slow recovery, both endoscopically and histologically, which supports the role of secukinumab as being the cause for the bowel pathology. Further control was achieved by the introduction of ustekinumab, which interferes with both IL-12 and IL-23 signaling.

Evidence points towards the implication of the IL-17/IL-23 axis in the pathogenesis in SLE [9] and its possible therapeutic application [10]. Clinical trials of IL-17 blockade with secukinumab in lupus nephritis is currently ongoing.

Therefore, given the potential risk of IBD exacerbation following treatment with IL-17 antagonists, one needs to consider evaluating patients for underlying bowel inflammation prior to treatment.

Our case highlights the need, when different immune-driven pathologies coexist, to find a therapeutic approach that could be beneficial for more than one disorder.

\section{Ethics and consent}

The patient has approved publication of his medical history.

\section{Acknowledgement and funding}

None.

\section{Conflict of interest}

None.

\section{References}

1. Petri M, Orbai AM, Alarcon GS, Gordon C, Merrill JT, et al. (2012) Derivation and validation of the Systemic Lupus International Collaborating Clinics classification criteria for systemic lupus erythematosus. Arthritis Rheum 8: 2677-2686. [Crossref]
2. Baeten D, Sieper J, Braun J, Baraliakos X, Dougados M, et al. (2015) Secukinumab, an Interleukin-17A Inhibitor, in Ankylosing Spondylitis. The New England Journal of Medicine 26: 2534-2548

3. Burisch J, Eigner W, Schreiber S, Aletaha D, Weninger W, et al. (2020) Risk for development of inflammatory bowel disease under inhibition of interleukin 17: A systematic review and meta- analysis. PLoS One 5: e0233781. [Crossref]

4. Gracey E, Vereecke L, McGovern D, Frohling M, Schett G, et al. (2020) Revisiting the gut-joint axis: links between gut inflammation and spondyloarthritis. Nature reviews Rheumatology 8: 415-433. [Crossref]

5. Fujino S, Andoh A, Bamba S, Ogawa A, Hata K, et al. (2003) Increased expression of interleukin

6. 17 in inflammatory bowel disease. Gut 53: 65-70.

7. Moschen AR, Tilg H, Raine T (2019) IL-12, IL-23 and IL-17 in IBD: immunobiology and therapeutic targeting. Nat Rev Gastroenterol Hepatol 3: 185-96.

8. Hueber W, Sands BE, Lewitzky S, Vandemeulebroecke M, Reinisch W, et al. (2012) Secukinumab, a human anti-IL-17A monoclonal antibody, for moderate to severe Crohn's disease: unexpected results of a randomised, double-blind placebo-controlled trial. Gut 12: 1693-1700. [Crossref]

9. Lee JS, Tato CM, Joyce-Shaikh B, Gulen MF, Cayatte C, et al. (2015) Interleukin-23Independent IL-17 Production Regulates Intestinal Epithelial Permeability. Immunity 4: 727-738

10. Zickert A, Amoudruz P, Sundstrom Y, Ronnelid J, Malmstrom V, et al. (2015) IL-17 and IL-23 in lupus nephritis - association to histopathology and response to treatment. BMC immunology 16: 7.

11. Van Vollenhoven RF, Hahn BH, Tsokos GC, Lipsky P, Fei K, et al. (2020) Maintenance of Efficacy and safety of ustekinumab through one year in a phase II multicenter, prospective, randomized, double-blind, placebo-controlled crossover trial of patients with active systemic lupus erythematosus. Arthritis Rheumatol 5: 761-768. [Crossref]

Copyright: (C2021 Almer S. This is an open-access article distributed under the terms of the Creative Commons Attribution License, which permits unrestricted use, distribution, and reproduction in any medium, provided the original author and source are credited. 